\title{
Vaccines for Papillomavirus (HPV) Infection
}

\author{
C. Rodríguez-Cerdeira ${ }^{*}, 1$ and A. Alba ${ }^{2}$ \\ ${ }^{I}$ Dermatology Department, CHUVI \& University of Vigo, Vigo, Spain \\ ${ }^{2}$ Centre for Molecular and Cellular Studies, Lugo, Spain
}

\begin{abstract}
Prophylactic HPV vaccination of both quadrivalent and bivalent vaccines has already been approved in Spain and is gaining popularity among Spanish women. Other European countries show similar trends.

With the use of vaccines against HPV, especially the tetravalent vaccine, in the short or medium-term there will be a lower rate of abnormal cytological results in vaccinated women, which is based on the expected preventive potential, which may range between 50 to $70 \%$ of the cases. Furthermore, with the use of the vaccine, in combination with present screening programs, we expect to reach a $92 \%$ reduction in the annual number of cases of cervical cancer and other associated pathologies in our country.
\end{abstract}

Keywords: HPV, bivalent vaccine, tetravalent vaccine.

\section{INTRODUCTION}

Persistent infection by human papillomavirus (HPV) is considered to be the principal causative agent in cervical cancer and other anogenital cancers. The demonstration that HPV-DNA is present in practically all cases of cervical cancer has a great importance to preventive strategies [1]. Thus, of the thirty genotypes known to infect the anogenital tract, it is estimated that worldwide HPV 16 and 18 cause $70 \%$ of the cervical cancers [1] and HPV 6 and 11 more than $90 \%$ of genital warts. The morbi-mortality and health costs associated with cervical cancer and its precursory lesions have provoked intense investigation over recent years $[3,4]$ in order to achieve prevention via vaccination against HPV, which would give a great opportunity to dramatically reduce the risk of this cancer [2].

Studies on animals demonstrated the immunogenic ability of particles which were self-assembled L1 proteins and were similar to viral particles. Data from clinical trials with three prototypes of prophylactic vaccines have demonstrated their effectiveness in the prevention of transitory and permanent incidental cervical infections caused by HPV 16 and 18 as well as in the cytologies and cervical lesions associated with the virus. With the immanent commercial launch of prophylactic vaccines for HPV, hope opens that we shall see a notable reduction, over the following years, in the rates of cervical cancer within the vaccinated populations. At the same time an intensive debate is taking place about the vaccination conditions (age, applicability, indication in men, composition according to the geographical area, necessity to vaccinate again,

*Address correspondence to this author at the Department of Dermatology, CHUVI, Meixoeiro Hospital \& University of Vigo, 36200 Vigo (Galicia), Spain; Tel: 00-34-600536114; Fax: 00-34-986-276416;

E-mails: aristoteles_cerdeira@yahoo.es; carmen.rodriguez.cerdeira@yahoo.es acceptance) cost-effectiveness, modification of the screening conditions for cervical cancer in developed countries and availability and accessibility of these in poorer countries.

\section{HPV INFECTION AND IMMUNITY}

Any viral infection requires the presence of a cellular receptor which allows the internalisation of the viral particles. This circumstance supposes the principal barrier to entry and explains the species-specific nature, and even organ-specific, of viral infections. Some viruses use the major histocompatibility complexes (MHC I and II), as receptors for their internalisation and others use molecules on the cellular surface (CD4, chemokines, growth factors, $\beta 2$ microglobulin. The HPV, do not have a specific cellular receptor but rather a well conserved surface molecule with vital cellular functions, which makes its use impossible as a target for blocking infection. As opposed to the case for other viruses, it does not appear that the surface receptors are implied in tissue or species specificity or in the tropism of the HPVs [3].

Both the recognition of the infection by the host cell and the specific tropism of each viral subgroup go to determine the cytopathic effects in specific tissues [4]; in this way, we distinguish between latent infections which do not show their effects for long periods of time, and active infections with practically immediate cytopathic effects. Based on these parameters it is possible to qualify the antigenic level or immunogenic level of each virus that would form the knowledge base for the manufacture of therapeutical or prophylactical vaccines.

\section{CELLULAR AND HORMONAL IMMUNITY TO HPV}

Cellular immunity is principally represented by T-cells which act at the level of the local tissues through close cell to cell contact. The humoral response is measured by the 
B-cells under instructions from the T-helper cells through the production of antibodies. The T-cells recognise proteins on the surface of the HPV associated with molecules from the cellular surface (HLA), whilst the antibodies do it both against surface antigens and soluble antigens. In the latter case this is done with greater specificity. The receptors of the T-cells recognise specific sequencies of small peptides presented by the MHC, whilst the antibodies recognise steric, three-dimensional structures with a determined structure. If the correct presentation of the antigen is essential for the induction of the immunological response, the kinetics of the antigen-antibody join, the number of these joins and their distribution, would be the factors which determine the level of the immunological response.

In general terms, after the first infection of the cervical epithelial cells by HPV, an unspecific response is provoked accompanied by an inflammatory process, chemoattraction of neutrophils, activation of macrophages, intervention of natural killer cells (NK), natural antibodies, and even the complementary system, which forms the first defensive unspecific immunological barrier. Prolongation of the response over time and protection against future infections requires specific immunological mechanisms.

The activation of the T CD4 lymphocytes requires the recognition of the surface molecules exposed by the presenter cell. The viral peptide together with the class II HLA, will be recognised in the context of TCR and CD4, but needs a "safety" mechanism to control the deactivation process. Thus it is necessary that other molecules such as CD40 and B7, present on the surface of the presenter cell, are recognised by their receptors (CD40 linking and CD28 respectively) for the activation to take place. The activated $\mathrm{T}$ CD4 lymphocyte will be converted into a type 1 or 2 lymphocyte $T$ helper cell, depending on a series of local tissue factors, fundamentally, antigen entry route, processing mechanism, and presence of different interleukins. The Th1 pathway will induce the maturity of the $\mathrm{T} C D 8+$ lymphocytes towards cytotoxic effector cells (Fig. 1).

Specific cells exist in the cervical epithelium which are capable of acting as antigen presenters and, although some keratinocytes develop this ability, it is Langerhans reticular cells which are the true specialists in antigen presentation in the cervical epithelium. These cells absorb the viral particles to digest them into endosomes and start an activation process which includes the presentation on the surface of the antigen together with the HLA of the presenter cells. These activated cells will be recognised by the T CD4 lymphocytes in the case that they recognise each and every one of the molecules in the correct environment, and, after that, evolve into lymphocyte helper (Th) in the local context of expression of certain interleukins (IL). Depending on the type of interleukin it will advance to differentiation towards a Th1 pathway that will induce the activation and proliferation of $\mathrm{T}$ CD8+ cytotoxins with specific immunity $(C T L+8)$ or it will advance towards a Th2 pathway that will induce the activation and expansion of B lymphocytes which develop differentiating towards plasma antibody producer cells for viral base proteins of unspecific immunity which we could identify as prophylactic.

The CTL +8 would have the ability to act against the established viral infection whilst the plasma B cells produce antibodies which act against the external viral antigens which are exposed during this and successive infections by HPV.

\section{PROPHYLACTIC VACCINES AGAINST HPV INFECTION}

Prophylactic vaccines against HPV infection, at present in an advanced stage of development and evaluation, seem to give more hope than therapeutic ones. They include products whose objective is to avoid new infections. Various approaches have been examined: recombinant live vector vaccines, protein and peptide vaccines, vaccines without DNA, and innocuous vaccines. The most advanced vaccines against HPV consist of particles similar to the virus called virus-like particles (VLP). They come from the capacities of the L1 and L2 proteins. These particles do not contain DNA and are synthesised through self-assembly of proteins of the upper antigen of the L1 capsid. VLPs are made by genetic engineering. They are structures which are identical to the native virus, but do not have infections capacity. The lower structural protein L2 can assemble with L1 and form an even more stable VLP. The antigenic similarities with the genuine virions of papilloma explain why VLPs introduce a powerful humoral response with neutralizing antibodies.

Gardasil $^{\circledR}$, tetravalent vaccine which includes VLPs of type $6(20 \mu \mathrm{g}), 11(40 \mu \mathrm{g}), 16(40 \mu \mathrm{g})$ and $18(20 \mu \mathrm{g})$ expressed in yeast cells of Sacchromyces cerevisiae

Fig. (1).

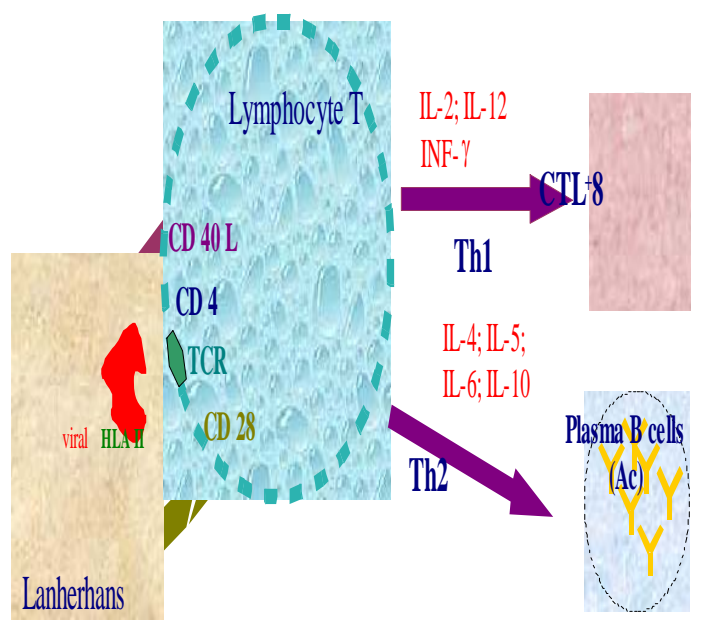


CANADE 3C-5 (stock 1895). It uses amorphous aluminium hydroxyphosphate sulphate as an adjuvant [5].

Cervarix ${ }^{\circledR}$, bivalent vaccine which includes VLPs of type $16(20 \mu \mathrm{g})$ and $18(20 \mu \mathrm{g})$ expressed in Baculovirus which uses Hi-5 Rix4445 cells derived from Trichoplusia ni. It uses adjuvant AS04 which is composed of aluminium hydroxide and MPL (3-0-desacyl-4'-monophophorile lipid A), a detoxified lipopolysaccharide obtained from Salmonella Minnesota [6].

Data have been published on the effectiveness for CIN $1 / 2 / 3$, AIS, VIN, VaIN and genital warts with a three year follow-up which reveals $95.5 \%$ to $100 \%$ effectiveness $[7,8]$.

In the short-term an appreciable impact of HPV vaccine is expected with a great reduction in abnormal cytological cervical results, which includes uncertain, squamous and glandular atypias, and LSIL, which are no more than the acute cytohistological response to viral presence, which is generally only temporary.

According to the Afrodita study, which reports on the prediction for preventive potential, of these pathologies, of tetravalent vaccine, Spain could avoid a total of 149,673 abnormal cytological results [9].

Moreover, the tetravalent vaccine also offers protection in the short-term from genital warts and probably against recurrent respiratory papillomatosis, caused by strains 6 and 11 of HPV [10].

The medium-term objective of these vaccines is the prevention of precursory lesions of cervical cancer, cervical intraepithelial neoplasia, and CIN, especially CIN 3 . Furthermore, many studies have been published in favour of protection against vulvar and vaginal neoplasias using the tetravalent vaccine [11].

The final objective in the long term of prophylactic vaccines for HPV is the prevention of invasive carcinoma of the cervix. Associated objectives are the prevention of other forms of cancer related to HPV: vulva, vagina, anus, penis and oropharynx [12].

In both vaccines it is highly important to guarantee the application of conservation protocols for cold storage, administration in the adequate anatomical area and recommended technique. We must also guarantee, the prevention of adverse reactions with post-vaccination vigilance.

Moreover, it is important to guarantee the registry in favour of fulfilling the whole vaccination plan. There is a very good safety profile for both vaccines within the age range evaluated in the tests 15-26 years old, and for the follow-up time available. This safety profile has been ratified until present with after sales vigilance, after the administration of millions of doses of Gardasil ${ }^{\circledR}$ and Cervarix ${ }^{\circledR}$ throughout the world $[12,13]$.

The test for HPV determination, which is more sensitive than cytology and highly specific when used within the right age group, has a profile which makes it suitable in vaccinated cohorts as a first screening test. Vaccinated women could, most probably, begin their controls later and at wider intervals: a screening strategy beginning at 30-35 years old with an interval of 5 years proves effective and efficient in the published models.

It is highly important that the vaccinated women do not perceive a false sensation of total protection against these pathologies. On the contrary, the vaccination should serve to detect a situation of inadequate or non-existent screening in vaccinated people or their direct families. We should vaccinate girls between 9 and 14 years old to prioritize the maximum preventive potential. Women up to $25 / 26$ years old should be vaccinated as the great preventive potential has been observed.

For boys between 9 and 14 years old, clinical tests with the vaccine Gardasil ${ }^{\mathbb{Q}}$ have warned us of the existence of greater immunogenicity in 9-15 year-old boys than in girls of the same age. We expect more conclusive results on effectiveness against transmission, persistent infection, genital warts and penile and anal lesions. Its importance also stems from the fact that it could be key from the public health point of view in case of achieving high coverage in women [14].

\section{THERAPEUTIC VACCINES AGAINST HPV}

Prophylactic vaccines will take various decades to show their impact, whilst a large percentage of infected women will have the risk of developing benign or malignant pathologies. It is, therefore, justified to investigate therapeutic vaccines capable of giving specific immunity mediated by the cells. The possible indications for the therapeutic vaccine are: 1) post-exposition, 2) diagnosis of low grade intraepithelial lesion (L-SIL), 3) diagnosis of high grade intraepithelial lesion (H-SIL) or invasive cancer [1517].

Data from models in dogs and rabbits make us think that vaccines which have the genes E1 and E2 as targets would be suitable for both post-exposed women and for those affected by L-SIL. Nonetheless, in women affected by CIN $2 / 3$ or cancer the continuous expression of the E6 and E7 oncogenes is essential for the progression and maintenance of the malignant phenotype. Experimental vaccines E6 and E7 have demonstrated immunogenicity and effectiveness, in terms of cure, in models of transplantable tumours in rodents. Nevertheless, trials in humans have demonstrated immunogenicity and safety but a very limited effectiveness $[16,18]$.

Therapeutic vaccines should be able to induce specific immunity mediated by cells capable of preventing the development of lesions and eliminating the existing lesions or even the malignant tumours. They use recombinant peptides derived from the oncogenes E6 and E7 (minigenes) [19-21].

\section{REFERENCES}

[1] Walboomers JM, Jacobs MV, Manos MM, et al. Human papillomavirus is a necessary cause of invasive cervical cancer worlwide. J Pathol 1999; 189: 12-6.

[2] Muñoz N, Bosch FX, de Sanjosé S, et al. Epidemiologic classification of human papillomavirus types associated with cervical cancer. N Engl J Med 2003; 348: 518-27.

[3] Muller M, Gissman L, Cristiano RJ, et al. Papillomavirus capsid binding and uptake by cells from different tissues and species. J Virol 1995; 69: 948-54. 
[4] Kadish A, Hagan RJ, Ritter DB. Biologic Characteristics of Specific Human Papillomavirus types predicted from morphology of cervical lesions. Hum Pathol 1992; 23: 1262-9.

[5] Block SL, Nolan T, Sattler C, et al. Comparison of the immunogenicity and reactogenicity of a prophylactic quadrivalent human papillomavirus (types 6, 11, 16, and 18) L1 virus-like particle vaccine in male and female adolescents and young adult women. Pediatrics 2006; 118: 2135-45.

[6] Giannini SL, Hanon E, Moris P, Van Mechelen M, Morel S, Dessy F. Enhanced humoral and memory B cellular immunity using HPV16/18 L1 VLP vaccine formulated with the MPL/aluminium salt combination (AS04) compared to aluminium salt only. Vaccine 2006; 24: 5937-49.

[7] Harper DM, Franco EL, Wheeler CM, et al. Sustained efficacy up to 4.5 years of a bivalent L1 virus-like particle vaccine against human papillomavirus types 16 and 18: follow-up from a randomised control trial. Lancet 2006; 367: 1247-55.

[8] Villa LL, Costa RL, Petta CA, et al. High sustained efficacy of a prophylactic quadrivalent human papillomavirus types 6/11/16/18 L1 virus-like particle vaccine through 5 years of follow-up. Br J Cancer 2006; 95: 1459-66.

[9] Puig-Tintoré LM, Castellsagué X, Torné A, et al. Coverage and factors associated with cervical câncer screening: Results from the AFRODITA study, apopulation-based survey in Spain. J Low Genit Tract Dis 2008; 12: 82-9.

[10] Tasca RA, Clarke RW. Recurrent respiratory papillomatosis. Arch Dis Child 2006; 91: 689-91.

[11] Cortés, J. Potencial preventivo a corto plazo de la Vacuna VPH. Impacto sobre los resultados citológicos anómalos. Available at http://www.aepcc.org (last accessed 5 April 2009).

[12] de Sanjosé S, Alemany L, Castellsagué X, Bosch FX. Human papillomavirus vaccines and vaccine implementation. Womens Health (Lond Engl) 2008; 4: 595-604.
[13] Rodríguez-Cerdeira C, Alba A, Vilata JJ. Desarrollo de nuevas vacunas contra el virus del papiloma humano. Piel 2007; 22: 51-3.

[14] Siegrist CA, Lewis EM, Eskola J, Evans SJ, Black SB. Human papilloma virus immunization in adolescent and young adults: a cohort study to illustrate what events might be mistaken for adverse reactions. Pediatr Infect Dis J 2007; 26: 979-84.

[15] Adams M, Borysiewicz L, Fiander A, et al. Clinical studies of human papilloma vaccines in pre-invasive and invasive cancer. Vaccine 2001; 19: 2549-56.

[16] Stanley MA. Progress in prophylactic and therapeutic vaccines for human papillomavirus infection. Expert Rev Vaccines 2003; 2: 381-9.

[17] Baldwin PJ, van der Burg SH, Boswell CM, et al. Vacciniaexpresed human papillomavirus 16 and 18 E6 and E7 as a therapeutic vaccination for vulvar and vaginal intraepithelial neoplasia. Clin Cancer Res 2003; 9: 5205-13.

[18] Adams M, Borysiewicz L, Fiander A, et al. Clinical studies of human papilloma vaccines in pre-invasive and invasive cancer Vaccine 2001; 19: 2549-56.

[19] Yugawa T, Kiyono T. Molecular mechanisms of cervical carcinogenesis by high-risk human papillomaviruses: novel functions of E6 and E7 oncoproteins. Rev Med Virol 2009; 19: 97 113.

[20] Hung CF, Ma B, Monie A, Tsen SW, Wu TC. Therapeutic human papillomavirus vaccines: current clinical trials and future directions. Expert Opin Biol Ther 2008; 8: 421-39.

[21] Bellone S, El-Sahwi K, Cocco E, et al. HPV16 VLP L1-specific CD8+ cytotoxic T lymphocytes (CTL) are equally effective as E7specific CD8+ CTL in killing autologous HPV16 positive tumor cells in cervical cancer patients: implications for L1 dendritic cellbased therapeutic vaccines. J Virol 2009; 83: 6779-89.

(c) Rodríguez-Cerdeira and Alba; Licensee Bentham Open.

This is an open access article licensed under the terms of the Creative Commons Attribution Non-Commercial License (http://creativecommons.org/licenses/by$\mathrm{nc} / 3.0 /$ ) which permits unrestricted, non-commercial use, distribution and reproduction in any medium, provided the work is properly cited. 